\title{
Planejamento e organização da atenção nutricional no enfrentamento do excesso de peso e da obesidade na Cidade do Recife-PE
}

Planning and organization of nutritional care in confromting excess weight and obesity in the City of Recife

Planificación y organización de la atención nutricional para afrontar el sobrepeso y la obesidad en la

Luana Yasmin Araujo Costa

ORCID: https://orcid.org/0000-0001-7968-0392 Centro Universitário dos Guararapes, Brasil

E-mail: luanayasminnutricionista@gmail.com

Fabiana Santos da Silva Oliveira ORCID: https://orcid.org/0000-0002-9686-3657 Faculdade de Ciências Humanas de Olinda, Brasil

E-mail: fabiana_san@hotmail.com

Rhayana Marques dos Santos ORCID: https://orcid.org/0000-0001-8820-091X Faculdade de Ciências Humanas de Olinda, Brasil E-mail: marquesrhayana@ hotmail.com

Vania Maria Silva de Moraes ORCID: https://orcid.org/0000-0002-8627-1690 Faculdade de ciências Humanas de Olinda, Brasil E-mail: vaniaenf_moraes@hotmail.com

Gleyce Kelly de Araújo Bezerra

ORCID: https://orcid.org/0000-0002-9388-7389

Universidade Federal de Pernambuco, Brasil E-mail: gleycearaujo.nutri@gmail.com

Maria Suzane da Silva Barbosa

ORCID: https://orcid.org/0000-0003-1995-8721

Universidade Federal de Pernambuco, Brasil E-mail: m.suzane.barbosa@gmail.com

Silvia Mariana da Silva Barbosa

ORCID: https://orcid.org/0000-0001-5252-9705

Universidade Federal de Pernambuco, Brasil E-mail: barbosasms@gmail.com

Danielle Cássia de Oliveira

ORCID: https://orcid.org/0000-0003-4154-2597

Faculdade Ciências Humanas de Olinda, Brasil E-mail: daniellecassiao@yahoo.com.br

\section{Resumo}

Objetivo: Este estudo buscou investigar a prevalência do excesso de peso e da obesidade, na cidade do Recife, analisar o processo de Planejamento e Organização da Atenção Nutricional no SUS, no combate ao excesso de peso e da obesidade e estimular o empoderamento para as práticas do autocuidado e da participação social. Metodologia: Utilizando método ecológico, de caráter analítico e abordagem qualitativa na perspectiva da intersetorialidade das Politicas Públicas de Alimentação e Nutrição, por meio de dados secundários e relatórios públicos do estado nutricional dos indivíduos acompanhados pelas equipes da Atenção Primária a Saúde cadastrada no SISVAN Web, indivíduos com idades entre 20 e 59 anos. Resultados: Observou-se que a prevalência do excesso de peso e da obesidade no município vem crescendo entre os anos de 2010 a 2019, em ambos os sexos, sendo mais frequente na população masculina. Considerações Finais: O conhecimento das realidades locais pode auxiliar em uma melhor definição de prioridades na agenda do governo. Palavras-chave: Gestão em saúde; sobrepeso e obesidade; Intersetorialidade; Segurança alimentar e nutricional.

\footnotetext{
Abstract

Objective: This study sought to investigate the prevalence of overweight and obesity in the city of Recife, to analyze the process of Planning and Organization of Nutritional Care in SUS, in combating overweight and obesity and to encourage empowerment for practices of self-care and social participation. Methodology: using an ecological method, with an analytical character and a qualitative approach from the perspective of the intersectoriality of Public Food and Nutrition
} 
Policies, using secondary data and public reports on the nutritional status of individuals monitored by Primary Care teams at Health registered at SISVAN Web, aged between 20 and 59 years. Results: It was observed that the prevalence of overweight and obesity in the municipality has been increasing between the years 2010 to 2019, in both sexes, being more frequent in the male population. Final Considerations: Knowledge of the local reality can help to better define the priorities of the government's agenda.

Keywords: Health management; Overweight And obesity; Intersectoriality; Food And nutrition security.

\section{Resumen}

Objetivo: Este estudio buscó investigar la prevalencia de sobrepeso y obesidad en la ciudad de Recife, analizar el proceso de planificación y organización de la atención nutricional en el SUS, en la lucha contra el sobrepeso y la obesidad y fomentar el empoderamiento para las prácticas de autocuidado. y participación social. Metodologia: Utilizando un método ecológico, con carácter analítico y un enfoque cualitativo en la perspectiva de la intersectorialidad de las Políticas Públicas en Alimentación y Nutrición, utilizando datos secundarios e informes públicos sobre el estado nutricional de los individuos monitoreados por los equipos de Atención Primaria de Salud registrados en SISVAN Web, envejecido entre 20 y 59 años. Resultados: Se observó que la prevalencia de sobrepeso y obesidad en el municipio ha ido aumentando entre los años 2010 a 2019, en ambos sexos, siendo más frecuente en la población masculina. Consideraciones finales: El conocimiento de las realidades locales puede ayudar a definir mejor las prioridades en la agenda del gobierno.

Palabras clave: Manejo de la salud; Sobrepeso y obesidad; Intersectorialidad; Seguridad alimentaria y nutricional.

\section{Introdução}

A Obesidade é um dos principais desafios de saúde pública da atualidade, estando presente nos países desenvolvidos e em desenvolvimento, embora a fome, a desnutrição e a falta de micronutrientes ainda demandem cuidados e investimentos do setor saúde, é alarmante o crescimento da prevalência da Obesidade e do excesso de peso (Opas, 2019).

O excesso de peso é determinado pelo aumento exclusivo de peso corporal, já a obesidade é definida como uma enfermidade crônica não transmissível (DCNT), multifatorial caracterizada pelo acúmulo excessivo de gordura que pode representar danos à saúde do indivíduo, além de predispor ao risco do desenvolvimento de outras patologias crônicas como a Dislipidemia, o Diabetes Mellitus (DM) e a Hipertensão Arterial (HAS) (Warmling, 2019; Abeso, 2016). Ou seja, trata-se de uma doença, e ao mesmo tempo um fator de risco, que demanda atuação multiprofissional e intersetorial no enfrentamento e no cuidado integral às pessoas acometidas, respeitando-se os princípios e diretrizes da Atenção Primária a Saúde (APS) (MS, 2020). Sendo o Nutricionista o grande articulador das ações de nutrição, juntamente com os demais profissionais (CFN, 2015).

A Atenção Primária a Saúde é a principal porta de entrada do SUS, por comunicar-se com toda rede de atenção, é um excelente espaço para ações de promoção, prevenção e recuperação da saúde da população. No âmbito federal o Ministério da Saúde (MS) subsidia os profissionais da APS no cuidado e diagnóstico da pessoa obesa, com publicações de material de apoio, programas e investimentos na educação permanente por meio de plataformas como a da Universidade Aberta do SUS (UNASUS) que dispõe de cursos gratuitos voltados para a temática e a elaboração anual de Protocolos Clínicos e Diretrizes Terapêuticas (PCDT) (MS, 2019; 2020).

O diagnóstico habitual da obesidade é realizado a partir da mensuração do índice de massa corpórea (IMC), calculado por meio da divisão do peso pela altura ao quadrado $\left(\mathrm{kg} / \mathrm{m}^{2}\right)$, recebendo critérios de gravidade quando associados a riscos metabólicos e clínicos, sendo necessária a associação de outros métodos de avaliação que quantifiquem e analisem a distribuição da gordura corporal, além de considerar outros fatores como o sexo, a idade e o comportamento alimentar, visto que avaliados isoladamente cooperam em erros de diagnóstico (MS, 2020; Alvarenga, 2015).

A Situação de Saúde no município o Recife vem se modificando nos últimos cinquenta anos, seguindo as tendências das mudanças no perfil de adoecimento do Brasil e do mundo, justificadas pelos processos de transição demográficas, epidemiológicas, nutricionais e sociais, dentro de um sistema marcado por uma tripla carga de doenças (Lira, 2020; Passos, 2018).

Este cenário é consequência do sistema alimentar vigente no Brasil que repercute diretamente na segurança alimentar e 
nutricional (Opas, 2017), representada por mudanças no padrão de alimentação determinadas pelo consumo excessivo de alimentos industrializados e ultraprocessados de elevada densidade energética, bem como o declínio na ingesta de alimentos in natura como frutas, verduras e legumes, associados à diminuição do nível de atividade física relacionados ao ambiente e mudanças nos modos de vida, sendo os principais responsáveis pela crescente ocorrência da obesidade e de sua determinação social no que diz respeito ao abastecimento e acesso a alimentos saudáveis, podendo ser passíveis de intervenções e ações de prevenção no âmbito coletivo e individual (Brasil, 2014; 2014).

A lógica da organização territorial e intersetorial do Sistema único de saúde (SUS) é o atendimento das necessidades de saúde da população, individual e coletiva, indicadas pelo perfil epidemiológico, salienta-se também que para que se possa garantir a integralidade da atenção, tanto as ações multiprofissionais, como a incorporação de ações de alimentação e nutrição no âmbito da Atenção Básica $(\mathrm{AB})$ se faz necessária, num esforço convergente e complementar às demais ações que já vêm sendo implantadas pelos diversos programas públicos de saúde ofertados, em especial a Estratégia Saúde da Família (ESF) (CFN, 2015).

Com base nestas reflexões e na análise de dados epidemiológicos, a questão norteadora deste estudo foi: Como se dá a operacionalização do processo de Planejamento e Organização da Atenção Nutricional no SUS, no Combate ao Excesso de Peso e a Obesidade, na perspectiva da intersetorialidade?

Dado o contexto apresentado e diante deste novo desafio na agenda da saúde pública representada pelo aumento da obesidade e do excesso de peso associado ao aumento das doenças crônicas não transmissíveis a nível mundial, decorrentes de mudanças no padrão de morbidade, demográficos e nutricionais. A pesquisa focou-se no cenário da Atenção Primária a Saúde local, Recife.

O objetivo do estudo foi de valorizar a $\mathrm{AB}$ por ser a mais próxima da comunidade, também pela sua capacidade de orientar e prevenir a população dos agravos à saúde, utilizando e demonstrando o potencial de apoio à gestão representada pelo uso dos sistemas de informações em saúde (Pinto, 2018), bem como a importância da participação social nos espaços deliberativos como expressão do empoderamento nas decisões públicas e avanço nas agendas da saúde, buscando contribuir com novos estudos que fomentem auxiliar na construção de um SUS onde a política intersetorial seja prioritária, que saia do discurso técnico e permaneça no planejamento orçamentário, ultrapassando a barreira da política partidária e focando na necessidade da população.

\section{Metodologia}

\section{Tipo de pesquisa}

Trata-se de um estudo ecológico de abrangência municipal, caracterizando-se como um estudo analítico de abordagem qualitativa (Amadei, 2011), que se utilizou de dados secundários concernentes à saúde pública, obtidos por meio de Sistemas de Informação em Saúde (SIS) (Pinto, 2018, Carvalho, 1998).

\section{Coleta e analise de dados}

Foram coletados os dados consolidados do SISVAN Web no período compreendido entre os anos 2010 a 2019. Para a análise estatística, foi utilizado planilha de Excel 2010.

Somado a isto, também foram realizadas consultas na base de artigos científicos indexados - Scientific Eletronic Library Online (Scielo), - utilizando as palavras-chave de forma isolada ou combinada: Planejamento e Gestão em Saúde; Excesso de peso e Obesidade; Redes de Atenção e Nutrição e Intersetorialidade, também foram analisados documentos e publicações disponíveis em sites oficiais do governo e de organizações ligadas ao enfrentamento da Obesidade no Brasil e no 
mundo, bem como em sites oficiais locais, acrescentados também dados de Inquéritos Populacionais, favorecendo uma visão ampliada da problemática da Obesidade reconhecida como um problema social e de Saúde Pública.

\section{População estudada}

A população do estudo foi constituída por adultos de faixa etária entre 20 e 59 anos, com diagnóstico de sobrepeso ou obesidade com ou sem morbidades, sendo incluídos os usuários da atenção primária a saúde (APS), SISVAN Web e os beneficiários do sistema de transferência de renda (Bolsa família).

\section{Critérios de classificação do estado nutricional}

Para estabelecer o diagnóstico nutricional utilizou-se a classificação do Índice de Massa Corporal (IMC) de acordo com o SISVAN utilizado na população brasileira e adaptado dos pontos de corte da Organização Mundial da Saúde (OMS) com IMC entre 25,0 e $29,9 \mathrm{Kg} / \mathrm{m}^{2}$ : excesso de peso; IMC entre 30,0 e $34,9 \mathrm{Kg} / \mathrm{m}^{2}$ : obesidade grau I; IMC entre 35,0 e 39,9 $\mathrm{Kg} / \mathrm{m}^{2}$ : obesidade grau II e IMC maior do que $40,0 \mathrm{Kg} / \mathrm{m}^{2}$ : obesidade grau III, com base nas recomendações da OMS, 2000 (Organização Mundial da Saúde).

\section{Categorias e variáveis}

As variáveis analisadas foram categorizadas da seguinte forma: No SISVAN Relatório do estado nutricional, fase da vida adulta 20 a 59 anos, Escolaridade (Todas); sexo (Feminino e Masculino); No Sistema de Nota Técnica do departamento estratégia saúde da família (DESF), consulta das equipes da Estratégia Saúde da Família (ESF) e CNES (Cadastro Nacional dos Estabelecimentos de Saúde), consulta das unidades básicas de saúde com cadastro no SISVAN.

\section{Procedimentos éticos}

Por se tratar de pesquisa que se utiliza de dados de domínio público, onde não há identificação dos indivíduos, não se faz necessária a submissão do projeto ao Comitê de Ética em Pesquisa, segundo as normas regulamentares de pesquisa envolvendo seres humanos, Resolução no 466 de 12 de Dezembro de 2012.

\section{Resultados}

Diante da coleta, analise e representação gráfica dos dados obtidos por meio de relatórios públicos do estado nutricional dos indivíduos acompanhados pelas equipes de Atenção Primária a Saúde no município de Recife, com idade entre 20 e 59 anos e classificados por sexo, disponíveis no sistema SISVAN-WEB, integrado aos sistemas de gestão da bolsa família e ao e-SUS $\mathrm{AB}$.

Segundo o Gráfico 1 da evolução do sobrepeso por sexo em Recife, foi observado que os homens só passaram a ter registro de sobrepeso a partir de 2011, tiveram uma frequência de mais anos com excesso de peso em relação as mulheres ou equivalente como no ano de 2015, exceto em 2014 onde as mulheres apresentaram 35\% e os homens $23 \%$ de peso elevado, um cenário favorável para as práticas preventivas de educação e atenção nutricional. 
Gráfico 1 - Evolução do Sobrepeso por sexo no município de Recife, nos anos de 2010 a 2019.

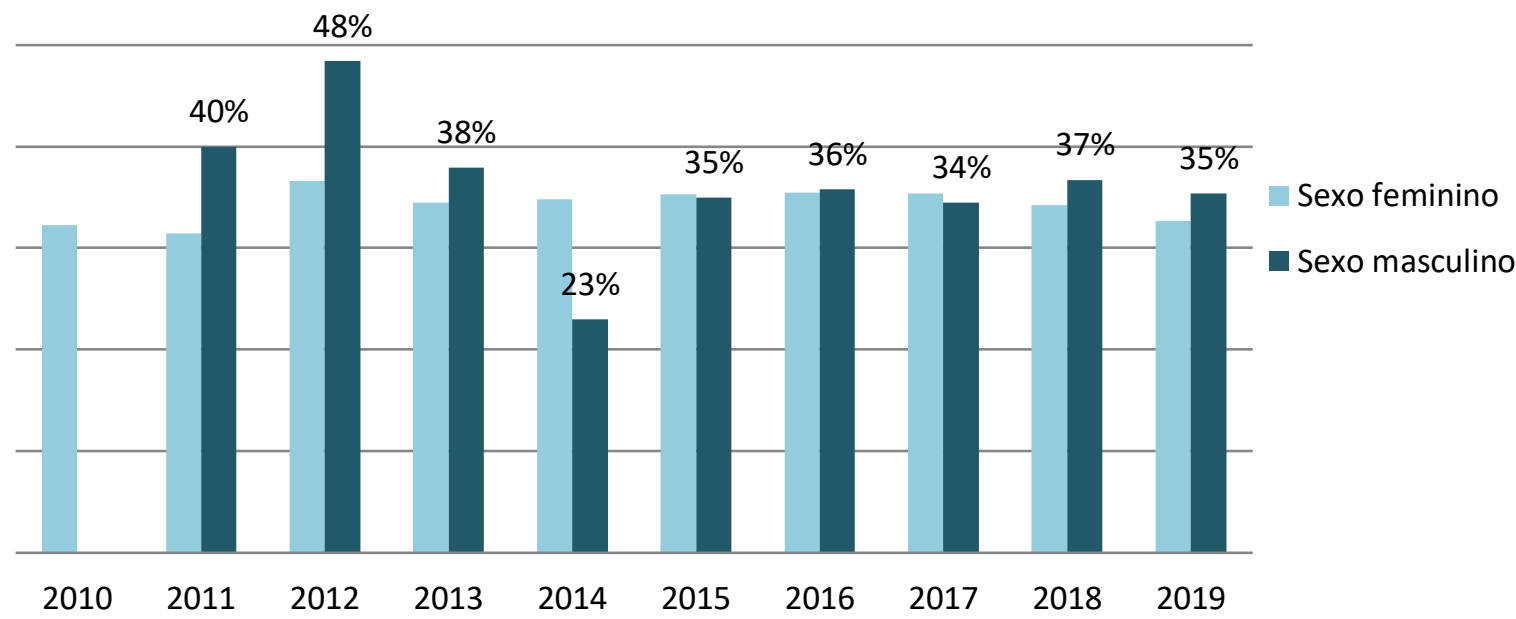

Fonte: SISVAN-WEB, e-SUS AB, Sistema de Gestão da Bolsa Família (DATASUS).

O Gráfico 2 demonstra a evolução da obesidade em homens, no qual o ano de 2010 foi excluído, pois não apresentou registro nos sistemas de informação da AB. Entre os anos de 2011 à 2019, a prevalência é de obesidade grau I e destacasse 2017 com percentual de 25\%, houve evolução de graus da Obesidade entre os anos de 2015 à 2019, onde 2015 apresenta os maiores índices de Obesidade Grau II com 10\% e Grau III aproximadamente 6\%, com tendência de alta da grau II, para os próximos dois anos.

Gráfico 2 - Evolução da Obesidade em graus em homens de 20 à 59 anos no município de Recife, entre 2011 e 2019.

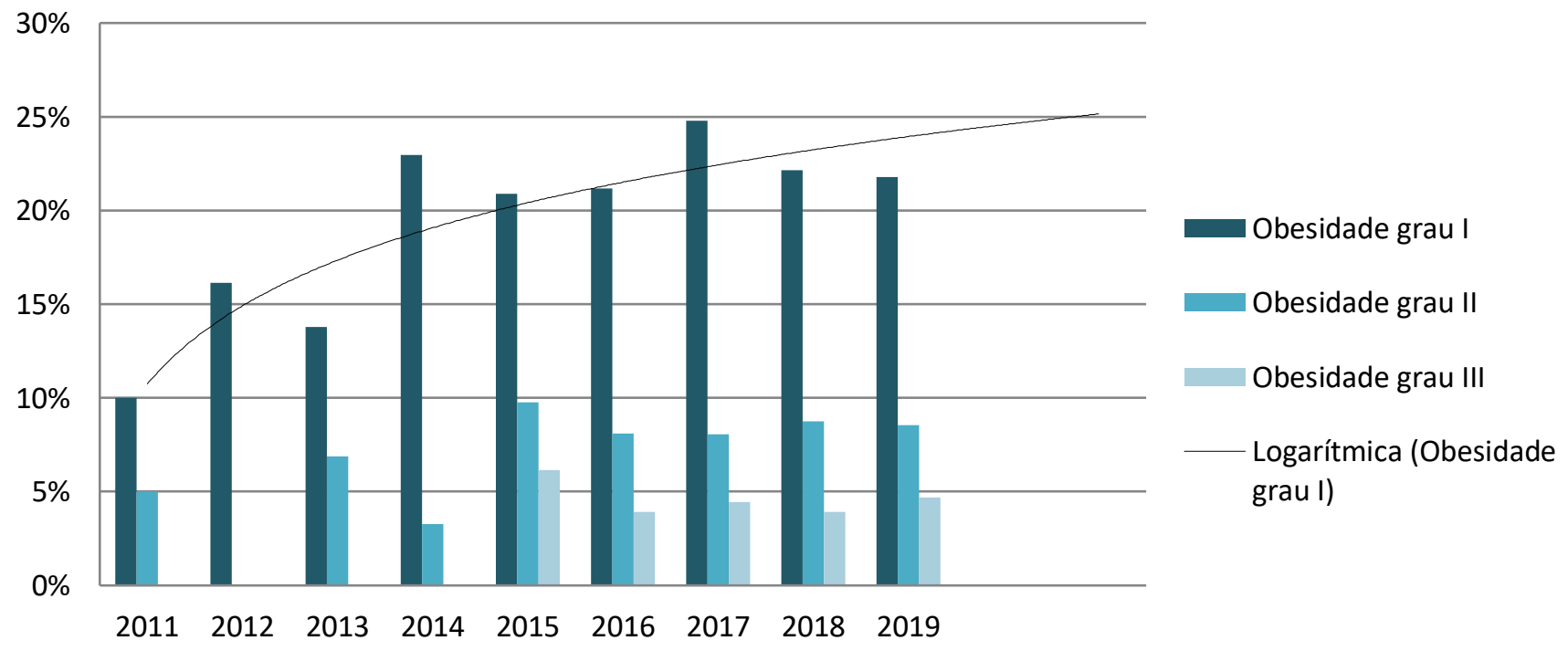

Fonte: SISVAN-WEB, e-SUS AB, Sistema de Gestão da Bolsa Família (DATASUS).

De acordo com o Gráfico 3 abaixo, destacasse a predominância da Obesidade grau I em crescimento no grupo das mulheres, e uma tendência de queda para os próximos dois anos, os graus I e II tiveram um aumento de $8 \%$ e $6 \%$ respectivamente entre o primeiro ano avaliado e o mais recente, demonstrando a necessidade de reestruturação na atenção e 
cuidado desta população na Atenção Básica.

Gráfico 3 - Evolução da Obesidade em mulheres de 20 à 59 anos no município de Recife entre 2010 e 2019.

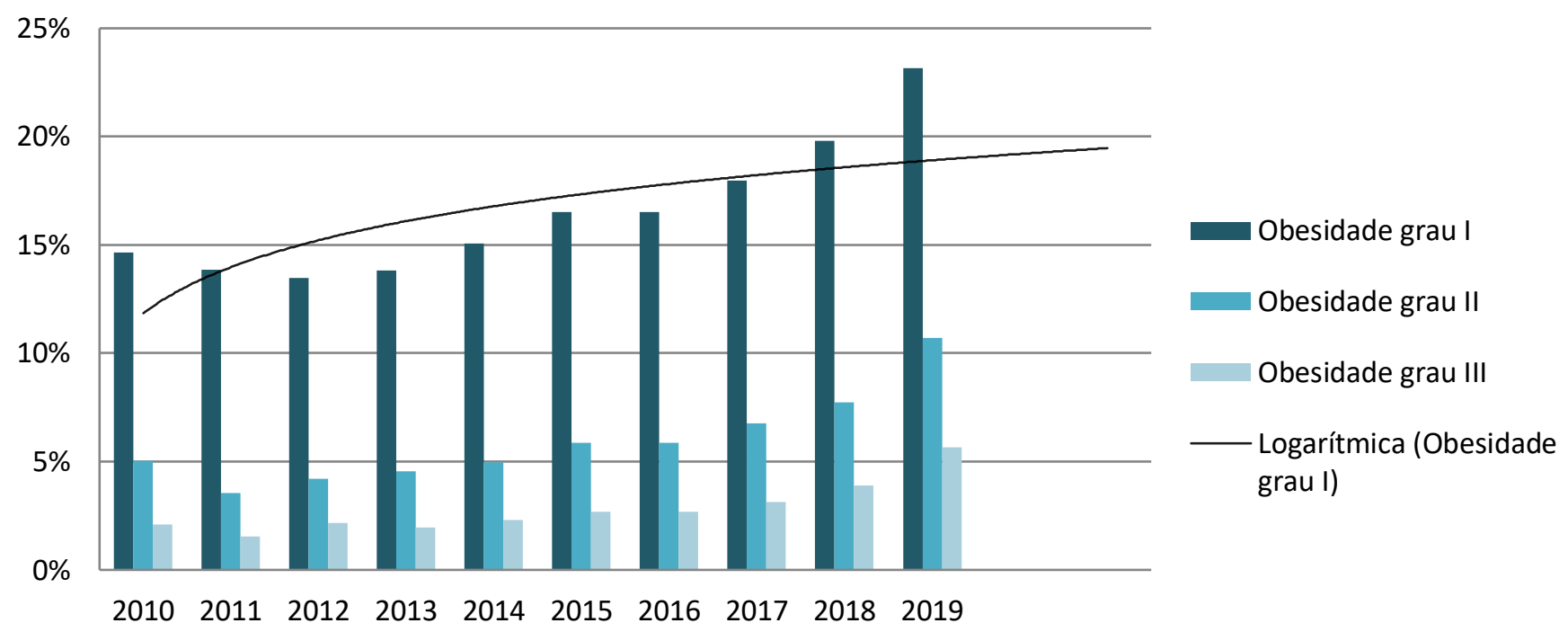

Fonte: SISVAN-WEB, e-SUS AB, Sistema de Gestão da Bolsa Família (DATASUS)

\section{Discussão}

Diante das descobertas demonstradas neste estudo, constata-se que o conhecimento da divergência social existente no território entre as áreas nobres e de baixa renda, auxilia na compreensão em partes dos altos índices de excesso de peso e obesidade encontrados nas UBS das áreas mais carentes, e também no planejamento de ações de combate aos agravos nutricionais locais, devendo considerar a natureza multifatorial da doença (Prefeitura do Recife, 2018).

O Sistema de Vigilância de Fatores de Risco e Proteção para Doenças Crônicas por Inquérito Telefônico (VIGITEL, 2006; 2019), revelou que mais da metade da população, aproximadamente 55,4\% está acima do peso em 2019. Um aumento de $30 \%$ se comparado com o percentual de $42,6 \%$ no ano de 2006. Em contrapartida Recife segundo dados do Sisvan Web indica os percentuais de 34,29\% em 2018 e 32,91\% em 2019.

Na capital Pernambucana de acordo com o VIGITEL (2018; 2019), conclui-se que a frequência de excesso de peso em mulheres cresceu de 22,6\% em 2018 para 23,4\%, em 2019, diferente do que foi encontrado no estudo atual no âmbito da atenção básica, onde os homens apresentam excesso de peso maior representado por 37\% em 2018 (pop. Total de 1.278) para 35\%, em 2019 (pop. Total de 1.570) e as mulheres 34\% em 2018 (pop. Total de. 28.740) para 33\%, em 2019 (pop. Total de. 17.866), porém, em um comparativo com os dados do VIGITEL, as mulheres apresentam frequências maiores de excesso de peso em relação à média populacional do município para o mesmo sexo.

Foi percebido que o percentual relevante de excesso de peso exposto chegou próximo do apresentado pela POF (2008 2009), que identificou excesso de peso em aproximadamente metade da população adulta, a PNS (2019) apresenta 60,3\% da população adulta, outros estudos populacionais locais como o de (Pinho, 2011), mostram claras tendências regionais e temporais do aumento progressivo da obesidade no país e no estado de Pernambuco, em seus resultados apresentou uma prevalência de $51,1 \%$ de excesso de peso na população Pernambucana.

A maior prevalência do excesso de peso observada entre os Homens investigados nesse estudo poderia ser atribuída a alguns fatores como uma população menor registrada nos relatórios do SISVAN Web, não sendo possível relacionar as 
causalidades como escolaridade, tipo de povo ou comunidade devido aos baixos registros, conforme demonstrado na literatura à idade tanto em homens como em mulheres dos 30 aos 39 anos predispõe ao ganho de peso, a maior escolaridade é considerada um fator de proteção e a menor de risco (Brasil, 2019).

Segundo dados do (Ibge, 2015) os homens apresentam menores frequências na prática de atividades físicas, consomem menos verduras, saladas e frutas, já o consumo de cerveja e bebidas alcoólicas é maior entre eles.

Apesar de a obesidade ser um problema de saúde mundial, conhecer as prevalências de sobrepeso e obesidade por regiões, estados e por municípios permite uma melhor definição de prioridades, assim como o planejamento de ações de forma regionalizada (Brasil, 2014).

No Brasil, as doenças crônicas aumentaram 67,8\% nos últimos treze anos, saindo de 11,8\% em 2006, para 19,8\% em 2018. Diante desta prevalência, vale ressaltar que a obesidade voltou a crescer (Brasil -VIGITEL, 2019). De acordo com Silva (2016) a obesidade é considerada fator de risco para diversas morbidades como o Diabetes, a Hipertensão, Doenças Cardiovasculares, dentre outras, apresentando a presença da obesidade como um determinante da saúde.

Conter o crescimento e reduzir as prevalências de sobrepeso e obesidade exige a articulação de diversas ações com níveis de complexidade diferentes, envolvendo mudanças sustentáveis nos ambientes e modos de vida e da população, bem como no papel que o SUS deve desempenhar dentro desse contexto (Brasil, 2014).

Segundo os achados deste estudo em relação à obesidade, verifica-se que esta esteve mais prevalente nas mulheres no conjunto de anos, embora a tendência temporal para os próximos dois anos seja de queda, entre o primeiro e o último ano houve aumento de mais de 5\% neste grupo, o fato destes valores não alcançarem a média dos estudos nacionais, não descaracteriza a obesidade como um problema crescente de saúde pública na cidade, demonstrando a importância e a necessidade de educação nutricional no âmbito das ações de promoção a saúde da Atenção Básica local.

De acordo com Eickemberg (2020), em um estudo com participantes do estudo longitudinal de saúde do adulto (ELSABrasil) com idades entre 35 e 74 anos, nos seus resultados a obesidade abdominal estratificada por cor, raça e sexo, está mais prevalente em Homens Brancos 69,9\% e uma frequência menor em mulheres pardas e pretas $63,3 \%$ e 60,0\%, respectivamente, em relação ao IMC dos autodeclarados pretos, apresentaram maiores valores em ambos os sexos.

Ferreira (2019) pontua que existem diferentes maneiras de mensuração da obesidade, sendo o índice de massa corporal (IMC) o principal indicador na avaliação do estado nutricional em adultos, em estudos populacionais, uma alimentação não saudável e exercício físico insuficiente são principais fatores de risco para obesidade. Encontrou em seus resultados, que a obesidade foi de $16,8 \%$ entre os homens e $24,4 \%$ entre as mulheres. Se comparados por sexo, tanto a prevalência de excesso de peso como a obesidade foram superiores no sexo feminino.

De acordo com a PNS (2019), a obesidade foi observada em 21,8\% dos homens e em 29,5\% das mulheres adultos. O indicador foi mais elevado no sexo feminino, chegando a 38,0\% das mulheres com idade de 40 a 59 anos, em comparação com $30,0 \%$ dos homens no mesmo grupo de idade.

O Sistema único de saúde da SMS (Secretaria Municipal de Saúde) de Recife praticava a saúde dentro de um modelo assistencialista centrado no médico, que não superava as necessidades locais da população, adotando um novo modelo de atenção e gestão, intitulado o Recife em defesa da vida, dentro deste contexto coube aos distritos sanitários sua implantação (Almeida, 2012).

No contexto da Atenção básica do município observados nesta pesquisa, alguns indicadores demonstraram relativa melhoria, registra-se em 2018 um aumento das unidades básicas de saúde (UBS), de 2,2\% no estado de Pernambuco e de 0,6\% quando comparado ao ano anterior, o município de Recife ainda não atinge o parâmetro de 1 unidade a cada 10 mil habitante, mas, está próximo. Quanto às equipes de saúde da família tem $11 \%$ em atendimento, os Agentes comunitários (ACS), ainda 
insuficientes, preocupante para o objetivo de prevenção de agravos, um ponto positivo é que o Recife detém boa parte da população informada no DATASUS (Aries, 2019).

Mesmo com os avanços com a implantação do novo modelo de gestão e assistência à saúde em Recife, dentro da equidade; o acesso e a utilização dos serviços; o financiamento a qualidade da atenção; a situação de saúde e as condições de vida da população (Bastos, 2015), e da informatização de toda rede municipal em 2011 (Prefeitura Do Recife, 2012). Com os serviços direcionados aos diversos segmentos sociais, os resultados revelam que inicialmente houve distanciamento da realidade do que foi planejado e na operacionalização no município, decorrentes das mudanças de gestão, conflitos de interesse nas esferas de governo, que ora influenciaram positivamente, ora negativamente.

Conforme analise da PMS (2014-2017) do município, um conjunto de ações vem sendo realizadas desde 2014, a fim de reduzir o número de pessoas com sobrepeso e obesidade e ainda prevenir o ganho de peso na população, como a Distribuição de DVD com o filme "Muito Além do Peso", o Matriciamento das equipes de saúde para o desenvolvimento de atividades voltadas a adoção de hábitos saudáveis; Prevenção da Obesidade em escolas - Mudanças de hábitos alimentares, aliados ao exercício físico; Avaliação do estado nutricional dos escolares do Programa Saúde na Escola; Avaliação do Estado Nutricional das crianças beneficiárias do Programa Bolsa Família, por meio do Sistema de Vigilância Alimentar e Nutricional (SISVAN). O que demonstra o compromisso público com as Políticas de Segurança Alimentar e Nutricional.

$\mathrm{Na}$ lógica de uma politica de governança aliado a resultados e que permite a avaliação e monitoramento para apoio a gestão, mesmo diante de um aumento expressivo na quantidade de pessoas com excesso de peso e obesidade na população recifense, a temática da obesidade foi citada pontualmente no novo plano de municipal de 2018-2021, dentro da rede temática das doenças crônicas, nos determinantes da saúde (Atividade física, excesso de peso), como provável responsável pelos aumentos da DM e HAS no município, desta vez sem apresentar nenhuma atualização quantas às ações que estão sendo planejadas ou realizadas na cidade.

Diversos programas implementados pela SMS, de prevenção e controle das DCNTs, ganham espaço no município com destaque para a Academia da Cidade do Recife institucionalizada como política municipal de promoção da saúde pela Portaria $n^{\circ}$. 122/2006, de 28 de setembro de 2006. Favorecendo a articulação de outros programas da cidade, como a Comunidade Saudável, a Requalificação e Reapropriação dos Espaços Públicos: Espaço Público para Todos. O Combate a Violência e Defesa da Cidadania, para fortalecer e ampliar as ações conjuntas desenvolvidas no âmbito da Promoção da Saúde, contribuindo o diálogo com a comunidade e acesso aos equipamentos de saúde através das ações conjuntas da ESF E NASF (Cruz, 2013).

Vários estudos têm associado diversas razões para o ganho de peso e sua manutenção em diferentes populações, deve se considerar algumas limitações neste estudo no recorte dos dez anos demonstrados, como os entraves tecnológicos, representados pela incompletude da informatização na rede municipal da AB, finalizada em 2011, o período de implementação do SISVAN nas unidades de saúde e a inclusão de novas unidades básicas de saúde e usuários ao longo dos anos estudados, as subnotificações por dificuldades técnicas de operacionalizar o sistema informatizado e o tempo de integração dos sistemas SISAB (Sistema de Informação em Saúde da Atenção Básica) como centralizador nacional com o e-SUS AB e o SISVAN que foi iniciada em 2017, todos estes fatores impactam nos resultados obtidos (Prefeitura do Recife, 2012). Sendo 136 UBS a mais no sistema de 2010 a 2020.

Brandão (2020) pondera que a coleta de dados antropométricos realizados pela $A B$ é importante na prevenção e tratamento do excesso de peso e que a baixa adequação aos processos de trabalho na prática da VAN, se deve em partes, a falta de estrutura e equipamentos adequados.

Diante do exposto podemos inferir que caminhando em sentido oposto as evidências científicas produzidas por inquéritos e pesquisas populacionais, pactos internacionais e aos esforços empreendidos pelo Ministério da Saúde, as secretarias 
de saúde estadual e municipal, na proposição de campanhas para prevenção e tratamento do excesso de peso e da obesidade, incorporados em um modelo de Sistema Universal de Saúde (SUS) que é federalista cooperativo, tripartite com autonomia local, em uma convergência entre as instancias de pactuação do SUS, a participação social e de demais órgão públicos ou privados nacionais e internacionais relacionados diretamente ou indiretamente com o setor saúde de enfrentamento aos agravos nutricionais, a população brasileira enfrenta um crescente problema de excesso de peso e obesidade e das DCNTs, nos últimos anos.

Ao longo dos trinta anos (30) de SUS, muitos avanços aconteceram, porém, ainda temos muitos desafios a serem discutidos. A Política da Atenção Básica, a PNAN assim como as demais precisa ser revista periodicamente, acompanhando as modificações temporais, tanto nos modos de gerir o sistema, como no perfil de adoecimento da população, envelhecimento e nascimento.

O Brasil ao longo da sua história de constituição de Políticas Públicas sofreu grande influência em cada momento político e econômico do país, que em alguns períodos suprimiram a autonomia de estados e municípios. A inserção da agenda de alimentação e Nutrição, inicialmente tratada meramente como assistencialista foi marcada por retrocessos, descontinuidades e fragilidades, sendo ademais institucionalizada como Política de Estado.

O país conquistou a Política Nacional de Alimentação e Nutrição, uma política do conjunto dos profissionais da saúde, que se apresenta com propósito de melhorar a alimentação, a saúde e garantir a Segurança Alimentar e Nutricional da população, com vistas ao DHAA, abrigando o discurso da intersetorialidade já na sua primeira edição em 1999, que falava sobre a dupla carga de doença, hoje tripla, e do problema do excesso de peso e da obesidade que se instalava.

Diante de mudanças determinadas pelas transições demográficas, epidemiológicas e nutricionais e no modelo de sistema alimentar, pautado no agronegócio, a PNAN precisou se reposicionar, sendo atualizada em 2014, e atuando como um importante instrumento norteador da governabilidade dos profissionais da saúde, inclusive do Nutricionista que tem o papel articulador das ações de Atenção Nutricional, prioritariamente na APS, mas, também em outros pontos de atenção dentro da RAS, assim como em atendimento as suas diretrizes nasce um conjunto de programas intersetorias de promoção à saúde e alimentação saudável, proporcionando autonomia e autocuidado as pessoas, em um processo de educação nutricional, um diálogo entre os profissionais e a população dentro das realidades locais.

Em Recife, como foi visto neste estudo, homens e mulheres entre 20 e 59 anos, da base de dados do SISVAN Web, atendidos nas unidades básicas de saúde (UBS) do município nos últimos 10 anos, vem aumentando a prevalência do excesso de peso e da obesidade, o que influenciou o aumento e dificuldade no controle de outras doenças crônicas na capital Pernambucana, em especial o DM e a HAS, conforme relatado em sua PMS 2018-2021. O envelhecimento da população observado na pirâmide populacional, ao mesmo tempo que indica melhorias nas condições de vida e controle nas DCNTs, é um ponto de atenção no planejamento municipal nas políticas de saúde.

Os esforços da SMS, na implantação de Políticas de Segurança Alimentar, na implantação de políticas nacionais como a academia da saúde, de urbanização com vistas à melhoria dos espaços públicos de convivência no sentido de promover a prática de atividades físicas e de aproximar a população aos equipamentos públicos de saúde, melhoria dos processos de trabalho, no fortalecimento da $\mathrm{AB}$, a troca de experiências entre as regiões de saúde, o alinhamento da gestão municipal com os princípios do SUS, objetivam resolver este agravo.

No âmbito internacional a união de ministros e secretários de saúde dos países das regiões das Américas, culminou na construção de um instrumento marco das políticas de planejamento estratégico a Agenda 30 para o desenvolvimento sustentável, que engloba temas inconclusos dos Objetivos de desenvolvimento do milênio e da Agenda de saúde das Américas 2008-2017, a nova agenda que apresenta 17 objetivos, se baseia nas lições das anteriores e expressa a visão de desenvolvimento sustentável da 
saúde, nesta região (Opas\Oms 2017).

Considerando a diversidade social dos grupos populacionais em sua distribuição distrital, é necessário superar os entraves na falta da completa integração destas ações, como a qualificação da força de trabalho no acolhimento do paciente obeso, a adoção das ações de vigilância na rotina de trabalho que sofre com as subnotificações e a melhoria no debate horizontal entre os níves de atenção pela continuidade do cuidado e informatização dos serviços.

Neste sentido diante deste novo desafio na agenda da saúde pública no Brasil e no município do Recife, este estudo sem extenuar todo o conteúdo sobre a temática, mostra que a lógica da organização político territorial permite enxergar claramente as regiões, sua população e problemas locais, buscou também demonstrar a importância da Atenção Básica como organizadora dos fluxos de atenção, promotora do dialogo com a população dentro e fora os equipamentos de saúde e influenciadora na melhora nas condições de saúde local, visto que essa relação no município houve bastante aderência pelos usuários.

\section{Considerações Finais}

Este estudo demonstra que a ação do profissional de saúde dentro das políticas intersetoriais do SUS impacta na saúde da coletividade, que é preciso sair da zona de conforto e compreender as lógicas das pactuações e discussões que acontecem no SUS, só assim poderá empoderar suas ações ainda que não exatamente dentro dos espaços de decisão, mas contribuindo no avanço das agendas, dentro dos serviços locais e assim estimular o empoderamento dos usuários para o autocuidado.

Por fim, buscou trazer um novo olhar para o estigma social sofrido pelo paciente obeso, pontuando que as causas nem sempre são individuais, que independentemente do IMC, é necessário garantir a oferta de ações de promoção da alimentação adequada e saudável e atividade física, seja na UBS ou em outros espaços no território, e assim dar andamento no processo de planejamento da Atenção Nutricional no município. Ressalta-se também a necessidade de novos estudos que demonstrem a visão dos usuários e profissionais no Recife sobre a relação com a temática do enfrentamento do excesso de peso e da obesidade, do campo da gestão para o campo da assistência.

\section{Referências}

Abeso (2016). Associação Brasileira para Estudo da Obesidade e da Síndrome Metabólica. Diretrizes Brasileiras de Obesidade.(4a ed.), https://abeso.org.br/wpcontent/uploads/2019/12/Diretrizes-Download-Diretrizes-Brasileiras-de-Obesidade-2016.pdf .

Alvarenga. M (2015). Nutrição comportamental, Manole, 549 p.

Amadei. J. R. P., \& Ferraz. V. C. T. (2019) Guia para elaboração de trabalhos acadêmicos (Trabalhos de conclusão de curso): ABNT NBR 14724:2011. Bauru, $51 \mathrm{p}$.

Aries (2019). Agência Recife para Inovação e Estratégia. Prefeitura da Cidade do Recife RECIFE 500. Olinda-PE.

Bastos, P. F. (2015). Território e redes de atenção à saúde no Recife: relação entre a Atenção Básica e os serviços de maior complexidade assistencial do SUS. Tese (Doutorado em Geografia), Universidade Federal de Pernambuco, Recife, 2015. https://repositorio.ufpe.br/bitstr eam/123456789/1 5526/1/TESE\%20Priscila\%20Felix\%20Bastos.pdf.

Brandão, A. L., et al (2020). Estrutura e adequação dos processos de trabalhos no cuidado à obesidade na Atenção Básica brasileira. Saúde em Debate, 44(126), 678-693. https://doi.org/10.1590/0103-1104202012607

Brasil (2014). Ministério da Saúde. Estratégias para o cuidado da pessoa com doença crônica: obesidade. Cadernos de Atenção Básica, n. 38. Pag. 63- Brasília: Ministério da Saúde. Pg. 37. http://189.28.128.100/dab/docs/portaldab/publicacoes/caderno_38.pdf.

Brasil (2020). Ministério da Saúde. Cadastro Nacional de Estabelecimentos de Saúde. Departamento de Informática do SUS. Consulta Estabelecimento. http://cnes.datasus.gov.br/.

Brasil (2014). Ministério da Saúde. Guia Alimentar para a População Brasileira. (2a ed.), Ministério da Saúde. http://portalsaude.saude.gov.br/images/pdf/2014/ novembro/05/Guia-Alimentar-para-a-pop-brasiliera-Miolo-PDF-Internet.pdf.

Brasil (2014). Ministério da Saúde. Perspectivas e desafios no cuidado às pessoas com obesidade no SUS: resultados do Laboratório de Inovação no manejo da obesidade nas Redes de Atenção a Saúde/Ministério da Saúde, Organização Pan-Americana da Saúde. - Brasília: Ministério da Saúde. 116 p. il. - (Serie Técnica Redes Integradas de Atenção a Saúde, v. 10). http://ecos-redenutri.bvs. 
Brasil (2011). Ministério da Saúde. Secretaria de Atenção à Saúde. Departamento de Atenção Básica. Orientações para a coleta e análise de dados antropométricos em serviços de saúde: Norma Técnica do Sistema de Vigilância Alimentar e Nutricional - SISVAN. Brasília: Ministério da Saúde. https://bvsms.saude.gov.br/bvs/publicacoes/orientacoes_coleta_analise_dados_antropometricos.pdf.

Brasil (2014). Ministério da Saúde. Secretaria de Atenção à Saúde. Departamento de Atenção Básica. Estratégias para o cuidado da pessoa com doença crônica: obesidade / Ministério da Saúde, Secretaria de Atenção à Saúde, Departamento de Atenção Básica. - Brasília: Ministério da Saúde. 212 p. (Cadernos de Atenção Básica, n. 38). http://bvsms.saude.gov.br/bvs/publicacoes/estrategias_cuidado_doenca_cronica_obesidade_cab38.pdf.

Brasil (2019). Ministério da Saúde. Secretaria de Vigilância em Saúde. Vigitel Brasil 2018: vigilância de fatores de risco e proteção para doenças crônicas por inquérito telefônico: estimativas sobre frequência e distribuição sociodemográfica de fatores de risco e proteção para doenças crônicas nas capitais dos 26 estados

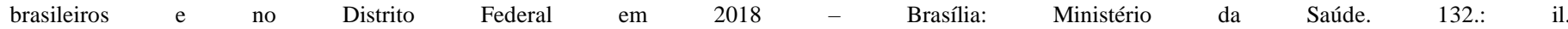
http://bvsms.saude.gov.br/bvs/publicacoes/vigitel_brasil_2018_vigilancia_fatores_risco.pdf.

Brasil (2007). Ministério da Saúde. Secretaria de Vigilância em Saúde. Secretaria de Gestão Estratégica e Participativa. Vigitel Brasil 2006: vigilância de fatores de risco e proteção para doenças crônicas por inquérito telefônico - Brasília: Ministério da Saúde. 297 p. : il. - (Série G. Estatística e Informação em Saúde). bvsms.saude.gov.br/bvs/publicacoes/vigitel_brasil_2006.pdf.

Brasil (2020). Ministério da Saúde. Secretaria de Vigilância em Saúde. Secretaria de Gestão Estratégica e Participativa. Vigitel Brasil 2019: vigilância de fatores de risco e proteção para doenças crônicas por inquérito telefônico - Brasília. http://bvsms.saude.gov.br/bvs/publicacoes/vigitel_brasil_2019.pdf.

Brasil (2020). Ministério da Saúde. Sistema de Nota Técnica do DESF. Departamento Saúde da Família - DSF. Coordenador Geral de Informação da Atenção Primária. Consulta Municipal, Relatório Municipal. http://sisaps.saude.gov.br/notatecnica/frmListaMunic.php.

Carvalho, A. de O. \& Eduardo. M. de P (1998). Sistemas de Informação em Saúde para Municípios, volume 6. São Paulo. Faculdade de Saúde Pública da Universidade de São Paulo. Série Saúde \& Cidadania.

CFN. Conselho Federal de Nutricionistas (2015). O papel do nutricionista na atenção primária à saúde. Recine E., Leão M., Carvalho M. F., [organização Conselho Federal de Nutricionistas]. (3a ed.), Brasília, DF: Conselho Federal de Nutricionistas. https://www.cfn.org.br/wp-content/uploads/2015/11/livretoatencao_primaria_a_saude-2015.pdf .

Cruz, D. K., Albuquerque, G., \& Damascena, W. Programa Academia da Cidade do Recife: minimizando contrastes sociais. Recife (PE): Portal DSS Nordeste. http://dssbr.org/site/experiencias/programa-academia-da-cidade-do-recife-minimizando-contrastes-sociais.

Eickemberg, M., et al (2020). Obesidade abdominal no ELSA-Brasil: construção de padrão-ouro latente e avaliação da acurácia de indicadores diagnósticos. Ciência \& Saúde Coletiva, 25(8), 2985-2998. https://doi.org/10.1590/1413-81232020258.20992018

Ferreira, A. P. de S., et al (2019). Prevalência e fatores associados da obesidade na população brasileira: estudo com dados aferidos da Pesquisa Nacional de Saúde, 2013. Revista Brasileira de Epidemiologia, 22, e190024. Epub April 01, 2019. https://doi.org/10.1590/1980-549720190024.

Ibge (2016). Pesquisa nacional por amostra de domicílios: síntese de indicadores 2015 / IBGE, Coordenação de Trabalho e Rendimento. - Rio de Janeiro: IBGE. 108p. https://biblioteca.ibge.gov.br/visualizacao/livros/liv98887.pdf.

Lira, P. I. C. (2020). Professor da UFPE coordena projeto de enfrentamento e cuidado da obesidade em PE. Iniciativa atende demanda do Ministério da Saúde e foi aprovado pelo CNPq junto com mais 22 projetos. Universidade Federal de Pernambuco - UFPE. https://www.ufpe.br/agencia/noticias//asset_publisher/VQX2pzmP0mP4/content/professor-da-ufpe-coordena-projeto-de-enfrentamento-e-cuidado-da-obesidade-em-pe/40615.

Ministério Da Saúde - MS (2019). Secretaria de Atenção Primária à Saúde- SAPS. Ministério da saúde lança carteira de serviços da Atenção Primária à saúdeBrasília (DF). https://aps.saude.gov.br/noticia/6694.

Ministério Da Saúde - MS (2020). Secretaria de Ciência, Tecnologia, Inovação e Insumos Estratégicos em Saúde - SCTIE. Departamento de Gestão e Incorporação de Tecnologias e Inovação em Saúde - DGITIS. Coordenação de Gestão de Protocolos Clínicos e Diretrizes Terapêuticas - CPCDT, elaboração da Comissão Nacional de Incorporação de Tecnologias no Sistema Único de Saúde - Conitec. Protocolo Clínico e Diretrizes Terapêuticas do Sobrepeso e Obesidade em Adultos - Brasília (DF). http://conitec.gov.br/images/Consultas/Relatorios/2020/Relatorio_PCDT_Sobrepeso_Obesidade_em_Adultos_CP_25_2020.pdf.

Opas (2019). Organização Pan-Americana da Saúde. O Panorama da Segurança Alimentar $e$ Nutricional. Brasília (DF). https://www.paho.org/bra/index.php?option=com_content\&view=article\&id=5799:desigualdade-exacerba-fome-desnutricao-e-obesidade-na-america-latina-e-nocaribe \&Itemid $=839$.

Opas (2017). Organização Pan-Americana da Saúde. Sistemas alimentares e nutrição: a experiência brasileira para enfrentar todas as formas de má nutrição. Brasília, DF: OPAS. https://www.cfn.org.br/wp-content/uploads/2017/09/oms.pdf.

Passos, R. S. (2018). Legislação do SUS, Saúde Pública e Epidemiologia para Concursos e Residências. João Pessoa, PB: Editora Brasileira \& Passos.

Pinho, C. P. S., et al (2011). Excesso de peso em adultos do Estado de Pernambuco, Brasil: magnitude e fatores associados. Cadernos de Saúde Pública, 27(12), 2340-2350. https://doi.org/10.1590/S0102-311X2011001200006.

Pinto, L. F, Freitas, M. P. S. de, F., \& André W. S. A. de. (2018) Sistemas Nacionais de Informação e levantamentos populacionais: algumas contribuições do Ministério da Saúde e do IBGE para a análise das capitais brasileiras nos últimos 30 anos. Ciênc. saúde coletiva, 23(6), 1859-1870. http://www.scielo.br/scielo.php?script=sci_arttext\&pid=S1413-81232018000601859\&lng=pt\&nrm=iso.

Pinto, L. F., et al (2018). Sistemas Nacionais de Informação e levantamentos populacionais: algumas contribuições do Ministério da Saúde e do IBGE para a análise das capitais brasileiras nos últimos 30 anos. Ciência \& Saúde Coletiva, 23(6), 1859-1870. https://doi.org/10.1590/1413-81232018236.05072018.

Pof (2011). Pesquisa de orçamentos familiares 2008-2009: análise do consumo alimentar pessoal no Brasil / IBGE, Coordenação de Trabalho e Rendimento. - Rio de Janeiro: IBGE. 150 p. Acompanha um CD-ROM, em bolso. 
Research, Society and Development, v. 10, n. 4, e15710413902, 2021

(CC BY 4.0) | ISSN 2525-3409 | DOI: http://dx.doi.org/10.33448/rsd-v10i4.13902

Prefeitura Do Recife (2018). Secretaria de Saúde. Plano Municipal de Saúde do Recife: 2018-2021. Recife. http://www2.recife.pe.go v.br/taxonomy/term/16627?op=MTMz.

Estevam. T. (2012). Recife comemora informatização de toda a Rede Municipal de Saúde. Prefeitura do Recife. http://www2.recife.pe.gov.br/noticias/12/09/2012/recife-comemora-informatizacao-de-toda-rede-municipal-de-saude.

Silva, E. C., et al (2016). Prevalência de hipertensão arterial sistêmica e fatores associados em homens e mulheres residentes em municípios da Amazônia Legal. Revista Brasileira de Epidemiologia, 19(1), 38-51. https://doi.org/10.1590/1980-5497201600010004.

Ward, J., et al (2015). Associação de fatores socioeconômicos com índice de massa corporal, obesidade, atividade física e fatores dietéticos em Belo Horizonte, Minas Gerais, Brasil: The BH Health Study. Cadernos de Saúde Pública, 31 (Supl. 1), 182-194. https://doi.org/10.1590/0102-311X00126914

Warmling, D, Araújo C. A. H, \& Sebold L. F. Reconhecendo o sobrepeso e a obesidade no contexto da atenção primária à saúde. Universidade Federal de Santa Catarina, 2019. $57 \mathrm{p}$ : il. www.unasus.ufsc.br. 